\title{
Prim and Floyd-Warshall Comparative Algorithms in Shortest Path Problem
}

\author{
Zuhri Ramadhan', Andysah Putera Utama Siahaan', M Mesran² \\ ${ }^{1}$ Faculty of Science and Technology, Universitas Pembangunan Panca Budi, Medan, Indonesia \\ ${ }^{2}$ Department of Computer Informatics, STMIK Budi Darma, Indonesia \\ ramadhanzoe@pancabudi.ac.id, andiesiahaan@gmail.com, \\ mesran.skom.mkom@gmail.com
}

\begin{abstract}
Optimization is an effort to obtain the best results by considering the constraints, constraints, and limitations that exist on a problem; the goal is to minimize or suppress the things that harm and maximize the things that are considered profitable. Generally, the Floyd-Warsall algorithm is widely used to solve shortest path problems, while the Prim algorithm is often used to solve the MST (Minimum Spanning Tree) problem in the Graph to minimize tree ranges or reduce the number of branches. Comparing the Prim algorithm with the Floyd-Warsall algorithm is expected to add proof if the Prim algorithm is not only used to solve Minimum Spanning Tree problem but can also solve the problem of Shortest Path Problem.
\end{abstract}

Keywords: Shortest Path, Prim, Floyd-Warshall, Optimation, Routing

\section{INTRODUCTION}

Security and speed are the most important of an algorithm [1]-[4]. It requires that an algorithm must be optimal [5]. Installation of electrical-related equipment also requires distance optimization to save raw material costs [6]. Many studies have raised the issue of optimization and the methods used to solve the problem. Just as in a network that has a path arrangement to connect multiple nodes. Familiar network models in community life such as road and toll road network systems[7], power lines and telephone and rail network. Optimization is an effort to obtain the best results by considering the constraints, constraints, and limitations that exist on a problem; the goal is to minimize or suppress the things that harm and maximize the things that are considered profitable [8] [9]. The term optimization can be related to the issue of the optimum route. The optimum route can be interpreted as the shortest route or the most efficient route from one place to the destination [10], but the two things are not the same, because the shortest route is not necessarily efficient and the most efficient route is not necessarily the shortest route. Viewed from the number of weight of each edge and the number of vertices of the vertex traversed. The smaller the number of weights $(\Sigma \mathrm{w})$ of the path through which the route is the shortest route even though the number of vertex dots $(\Sigma v)$ more. Apart from that the smaller the number of vertex dots $(\Sigma \mathrm{v})$ of the path through which the route is the most efficient route. Prim and Floyd-Warshall algorithms are algorithms that serve to find the shortest route [11], [12]. In general, the Floyd-Warsall algorithm is widely used to solve the shortest path problem (Shortest Path Problem). As research conducted by Akanksha Singh and Pramod Kumar Mishra [13] with the title "Performance Analysis of Floyd Warshall Algorithm vs. 
Rectangular Algorithm." The Prim algorithm is often used to solve the problem of Minimum Spanning Tree on the graph to minimize the range of trees or reduce the number of branching. The prim algorithm can also be used to find the shortest path with a minimal number of paths and smaller duration. Comparing the Prim algorithm with the Floyd-Warsall algorithm is expected to add proof if the Prim algorithm is used not only to solve the Minimum Spanning Tree problem but also to solve the Shortest Path Problem problem. This study tried to compare the two algorithms. The result of this research is which algorithm is better and optimal in finding the shortest route.

\section{THEORIES}

\subsection{Floyd-Warshall Algorithm}

Robert W. Floyd found this algorithm in 1967. Floyd-Warshall's algorithm has a directed and weighing directed graph $(\mathrm{V}, \mathrm{E})$, which is a point list (node / vertex V) and a side list (edge $\mathrm{E})$. The number of side weights on a path is the weight of the path. The side on $\mathrm{E}$ is allowed to have a negative weight, but it is not allowed for this graph to have a cycle of negative weights. This algorithm calculates the smallest weights of all paths connecting a pair of points and does so at the same time for all pairs of points.

The Floyd-Warshall algorithm compares all possible paths in the graph for each side of all nodes. It may be due to the estimation of decision making (shortest path selection) at each stage between two vertices until the estimate is known as the optimal value. Suppose there is a graph $\mathrm{G}$ with vertices $\mathrm{V}$, each numbered 1 to $\mathrm{N}$. There is also the shortest path function $(\mathrm{i}, \mathrm{j}, \mathrm{k})$ that returns the possibility of the shortest path from $i$ to $j$ using only the node 1 to $\mathrm{k}$ as an intermediate point. The ultimate purpose of this function is to find the shortest path from each node $i$ to $j$ with an intermediate node 1 to $k+1$. The implementation of the Floyd-Warshall algorithm can be seen in the following figure.

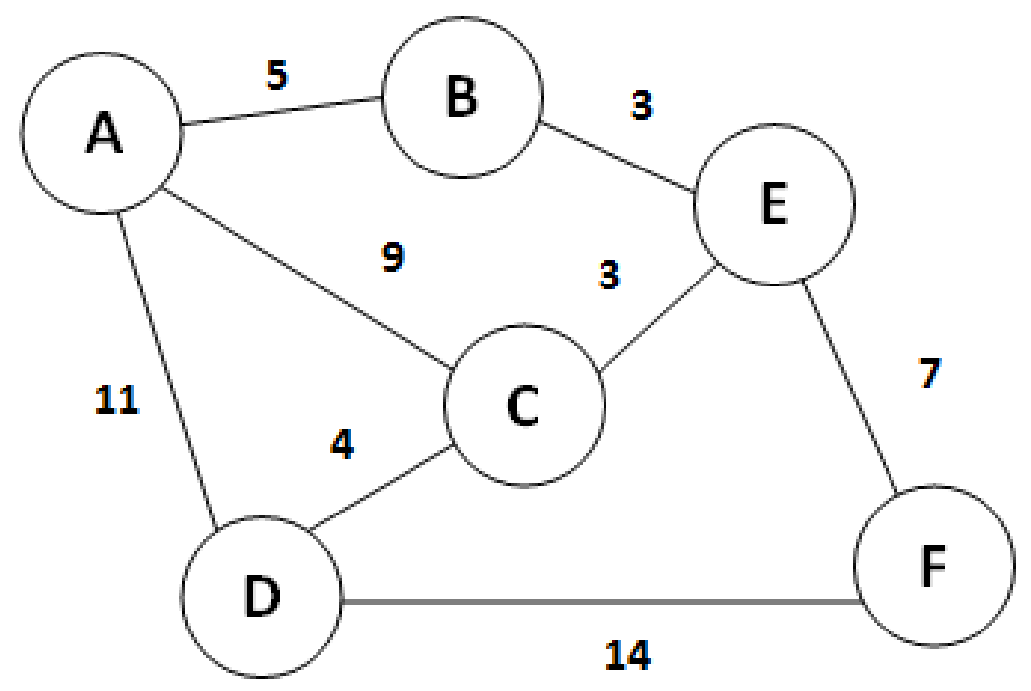

Figure 1. Floyd-Warshall graph 
There are several paths between $\mathrm{A}$ and $\mathrm{F}$ as shown in erlier figure.

Path $1:$ : A - B - E - F $=15$

Path $2:$ A - B $-\mathrm{E}-\mathrm{C}-\mathrm{D}-\mathrm{F}=29$

Path $3:$ : A - C $-\mathrm{E}-\mathrm{F}=19$

Path $4:$ A $-\mathrm{C}-\mathrm{D}-\mathrm{F}=27$

Path $5:$ A $-\mathrm{D}-\mathrm{F}=25$

The conclusions that can be taken from the above analysis are as follows:

- There can be more than one route between two nodes.

- The number of nodes in the route is not important (Line 1 has four nodes but is shorter than Line 5, which has three nodes).

- There can be more than one minimal length path.

\subsection{Prim Algorithm}

The algorithm was invented in 1930 by mathematician Vojtèch Jarnik and later separately by computer scientist Robert C. Prim in 1957 and rediscovered by Dijkstra in 1959 [14]. The prim algorithm is one algorithm that works in greedy. The Prim algorithm forms a step by step Minimum Spanning Tree. At each step, a graph side of $G$ has a minimum weight and is connected to the Minimum Spanning Tree that has been formed. The prim algorithm is used to find the minimum plant of the weighted connected graph by taking the side of the line having the smallest weight of the graph, where the line is side by side with the outstretched tree that has been created and which does not make the cycle [15]. An algorithm in graph theory is to find the minimum tree range for an interconnected weighted graph; it means a subset of edges that form a tree containing nodes, where the overall weight of all edges in the tree is of minimal value. If the graph is not connected, then the graph has only one minimum range tree for one of the connected components.

The following table describes the complete example of the Prim algorithm.

Table 1. Prim Algorithm example

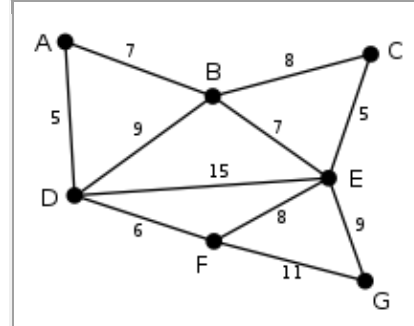

It is an initial weighted graph. This graph is not a tree because there is a circuit. A more precise name for this diagram is a graph or a network. The numbers near the connecting line are the weights. No lines have been marked, and node D is selected arbitrarily as a starting point. 


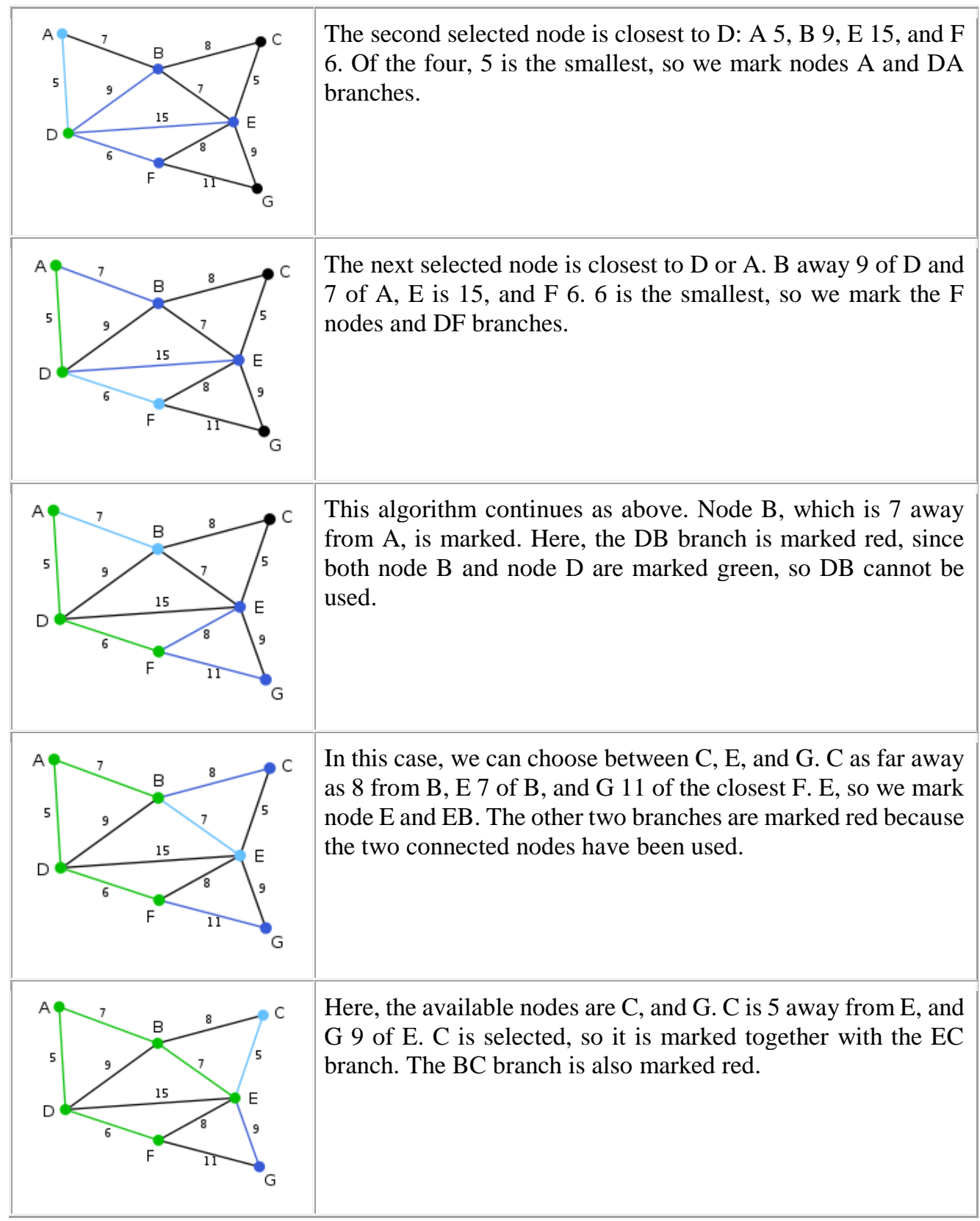




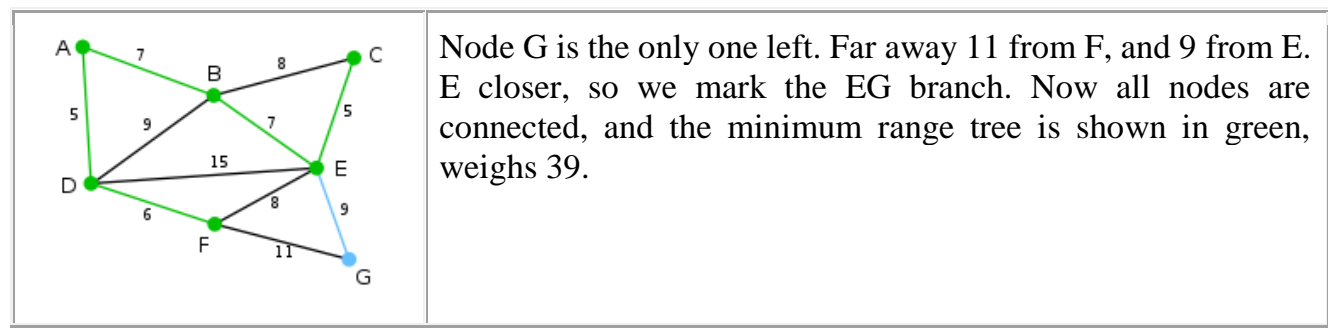

\section{LITERATURE REVIEW}

\subsection{Kalpanadevi's research}

D. Kalpanadevi's [16] research entitled "Effective searching for shortest paths in graphs using Prim's algorithm." This study discusses how to find the path for the shortest path when selecting the starting location by using a primer algorithm. It is commonly used in the minimum spanning tree to be connected to a weighted graph $\mathrm{G}(\mathrm{V}, \mathrm{E})$, to find a subset of edges by forming a tree that covers every point, where the total weight of all edges in the tree is minimized. The Prim algorithm is used to find the minimum shortest path distance between two locations; the user must decide, visit the place and reach the destination as their initial planning with time duration. From the execution, two results are taken for minimum road distance and highway to follow the route from the node to reach the goal efficiently. Under the result, a high road ramp is the best way to drive to reach the destination. The selection of the vertices depends on the graph itself. Finally, the Prim algorithm runs efficiently not only on the optimal path found in the graph and also runs effectively to find the optimal highway rather than the shortest path in a standard way of the path and also to find the shortest path of clear road traffic and reach the destination with on low period.

\subsection{Hougardy's research}

Hougardy's [17] research entitled "The Floyd-Warshall Algorithm on Graph with Negative cycles." This study discusses the Floyd-Warshall algorithm used to calculate the shortest path between all pairs of vertices on the side of a weighted and directed graph. It can also be used to detect the existence of negative cycles. This paper would like to try to prove that for this task many existing implementations of the Floyd-Warshall Algorithm will fail because large exponential numbers can appear during implementation. Conclusion: Hougardy has pointed out that during the implementation of Floyd-Warshall algorithms large quantities of exponentials may occur if the input graph cycle contains negatives. The second theory implies that even for a graph with less than 30 vertices and 60 sides weighing -1 it may be possible that during the implementation of the Floyd-Warshall algorithm with an absolute value greater than 264. It suggests that for larger graphs it can be quite likely to have sub graph that causes the width.

\subsection{Singh and Mishra's research}

Singh and Mishra's [13] research is titled "Performance analysis of Floyd-Warshall algorithms vs. rectangular algorithm." In this paper, they have examined the comparative study of the Floyd Warshall algorithm and the Rectangular algorithm. Singh and Mishra have tested both algorithms on a random graph generated by the Erdös - Renyi (ER) Model. Evaluation of 
algorithms for different probabilities suggests that the Floyd Warshall algorithm provides slightly better performance for stable graphs while Rectangular algorithms work better for sparse graphs.

\section{METHODOLOGY}

\subsection{Graph Design}

The graph used in this research is obtained by designing based on the coordinate axis $\mathrm{X}$ and Y. Coordinate axis is used to determine the weight between vertices by utilizing Euclidean Distance method. The graph to be used as a test sample of ten graphs, each of which has different weights and will be grouped into five levels. It is intended to obtain a more empirical proof. The following graph grouping data corresponds to the number of vertices that will be generated on each graph in the following table.

Table 2. List the Vertex Amount of Each Graph

\begin{tabular}{|c|c|c|c|c|}
\hline Level & Graph & Vertex & Edge & Notation \\
\hline \multirow{2}{*}{1} & G1 & 3 & 3 & A,B,C \\
\hline \multirow{2}{*}{ II } & G2 & 4 & 4 & A,B,C,D \\
\cline { 2 - 5 } & G3 & 4 & 5 & A,B,C,D \\
\hline \multirow{2}{*}{ III } & G4 & 5 & 6 & A,B,C,D,E \\
\cline { 2 - 5 } & G5 & 5 & 7 & A,B,C,D,E \\
\hline \multirow{2}{*}{ IV } & G6 & 6 & 8 & A,B,C,D,E,F \\
\cline { 2 - 5 } & G7 & 6 & 9 & A,B,C,D,E,F \\
\hline \multirow{3}{*}{ V } & G8 & 7 & 10 & A,B,C,D,E,F,G \\
\cline { 2 - 5 } & G9 & 7 & 11 & A,B,C,D,E,F,G \\
\cline { 2 - 5 } & G10 & 7 & 12 & A,B,C,D,E,F,G \\
\hline
\end{tabular}

\subsection{Graph Weighting}

Graph weighting is done by using the Euclidean distance method commonly used to calculate the length of the diagonal line on the triangle [18]. However, to obtain the distance of the diagonal line and the straight line parallel to the $\mathrm{x}$ and $\mathrm{y}$-axes, the vertex points should be presented in 2-dimensional coordinate form ( $\mathrm{x}, \mathrm{y})$ so that it can be calculated using the following formula.

$$
D=\sqrt{(x 2-x 1)^{2}+(y 2-y 1)^{2}}
$$

The ABS function is used when the starting point is farther away from the destination point (reverse), so the reduction of $\mathrm{x} 2-\mathrm{x} 1$ or $\mathrm{y} 2-\mathrm{y} 1$ produces a negative value. This function is to convert the negative value into the positive value. 


\section{RESULT AND DISCUSSION}

\subsection{Prim Test}

The Prim algorithm test will use the table for each graph step in finding the optimum route and minimize the active vertex. The test is done by using 10 sample graph with name G1, G2, G3, G4, G5, G6, G7 G8, G9, and G10. This test produces the optimum path with the shortest distance.

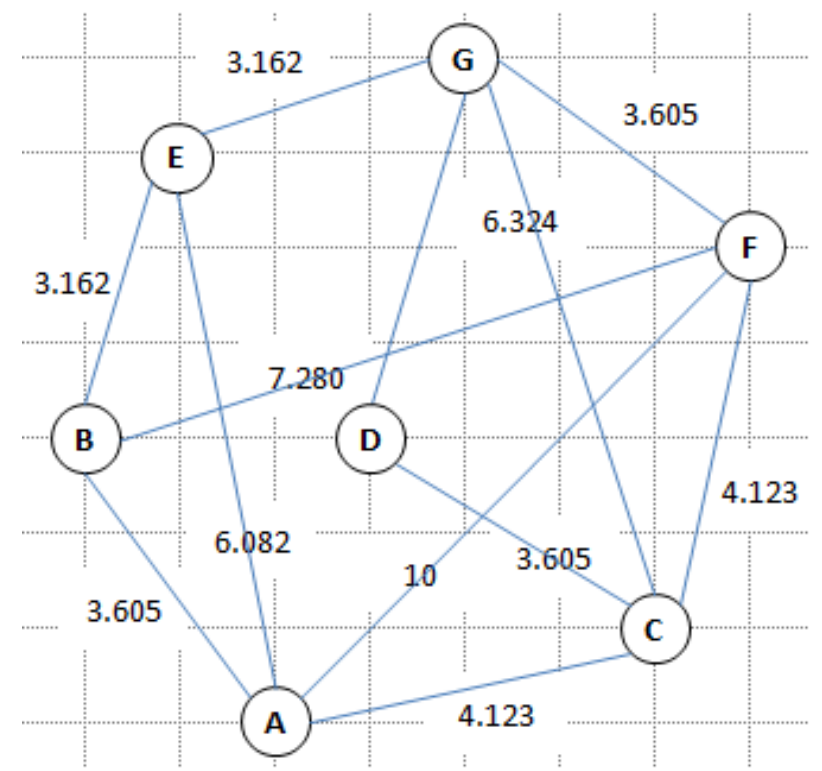

Figure 2. The final test sample (G10)

The following table shows the overall calculations of G1 to G10.

Table 3. Graph sample calculation result

\begin{tabular}{|c|c|c|c|}
\hline Graph & $\begin{array}{c}\text { Active } \\
\text { Vertex }\end{array}$ & Optimum Route & Cost \\
\hline G1 & 2 & $\mathrm{~A}-\mathrm{B}-\mathrm{C}$ & 9 \\
\hline G2 & 3 & $\mathrm{~A}-\mathrm{B}-\mathrm{D}$ & 9 \\
\hline G3 & 3 & $\mathrm{~A}-\mathrm{C}-\mathrm{D}$ & 7.285 \\
\hline G4 & 3 & $\mathrm{~A}-\mathrm{C}-\mathrm{D}-\mathrm{E}$ & 13.605 \\
\hline G5 & 2 & $\mathrm{~A}-\mathrm{D}-\mathrm{E}$ & 9.857 \\
\hline G6 & 3 & $\mathrm{~A}-\mathrm{C}-\mathrm{D}-\mathrm{F}$ & 11.423 \\
\hline G7 & 3 & $\mathrm{~A}-\mathrm{D}-\mathrm{E}-\mathrm{F}$ & 8.992 \\
\hline
\end{tabular}




\begin{tabular}{|c|c|c|c|}
$\mathrm{G} 8$ & 3 & $\mathrm{~A}-\mathrm{B}-\mathrm{E}-\mathrm{G}$ & 9.929 \\
\hline $\mathrm{G} 9$ & 3 & $\mathrm{~A}-\mathrm{B}-\mathrm{E}-\mathrm{G}$ & 9.929 \\
\hline $\mathrm{G} 10$ & 3 & $\mathrm{~A}-\mathrm{B}-\mathrm{E}-\mathrm{G}$ & 9.929 \\
\hline
\end{tabular}

\subsection{Floyd-Warshall Test}

Floyd-Warshall testing algorithm allows all points on the graph to be a starting point, but in this study point A becomes the starting point, and the point in the last alphabet becomes the endpoint. The test is done by using 10 sample graph with name G1, G2, G3, G4, G5, G6, G7 G8, G9, and G10. Testing aims to obtain the optimum path with the shortest distance.

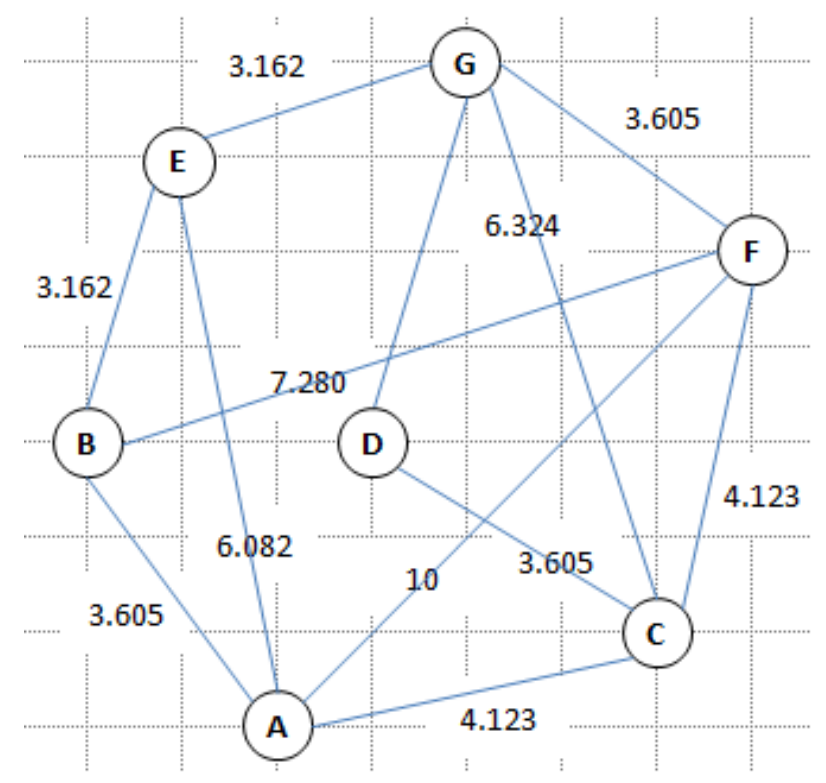

Figure 3. The final test sample (G10)

The following table shows the overall calculations of G1 to G10.

Table 4. Graph sample calculation result

\begin{tabular}{|c|c|c|c|}
\hline Graph & $\begin{array}{c}\text { Active } \\
\text { Vertex }\end{array}$ & Optimum Route & Cost \\
\hline G1 & 1 & A -C & 6.403 \\
\hline G2 & 2 & A - C - D & 8.099 \\
\hline G3 & 1 & A - D & 6.403 \\
\hline G4 & 2 & A - B - E & 11.181 \\
\hline G5 & 2 & A - B - E & 9.472 \\
\hline
\end{tabular}




\begin{tabular}{|c|c|c|c|} 
G6 & 2 & A - E - F & 8.992 \\
\hline G7 & 2 & A - C - F & 8.261 \\
\hline G8 & 2 & A-E - G & 9.244 \\
\hline G9 & 2 & A - E - G & 9.244 \\
\hline G10 & 2 & A-E - G & 9.244 \\
\hline
\end{tabular}

\subsection{Comparison of the two algorithms}

The test result of graph 1 to 10 on each algorithm produce optimum path traversed and cost. The calculation results can be seen in the following table.

Table 5. Active vertex raised comparison

\begin{tabular}{|c|c|c|}
\cline { 2 - 3 } \multicolumn{1}{c|}{} & Floyd & Prim \\
\hline G1 & 2 & 2 \\
\hline G2 & 2 & 3 \\
\hline G3 & 3 & 3 \\
\hline G4 & 4 & 4 \\
\hline G5 & 4 & 4 \\
\hline G6 & 4 & 5 \\
\hline G7 & 5 & 5 \\
\hline G8 & 6 & 6 \\
\hline G9 & 7 & 6 \\
\hline G10 & 8 & 6 \\
\hline SCORE & $\mathbf{4 , 5}$ & $\mathbf{4 , 4}$ \\
\hline
\end{tabular}

Table 5 shows the active vertices ratio generated by each algorithm. In graph 1 to 10 , each algorithm showing different performance in finding the optimum route by generating active vertex. To know which algorithm is more optimum in generating active vertex hence taken average from the result of the whole graph. If the average number of active vertices is taken in each graph, then the result obtained where the Floyd-Warshall get a mean value of 4.5 while for the Prim algorithm get the average value of 4.4. It means the primer algorithm is faster in determining the optimum route by generating the less active number of vertices. The comparison of the optimum path through which both algorithms can be seen in the following table.

Table 6. Comparison of vertex counts optimum lines

\begin{tabular}{|c|c|c|c|c|}
\hline & \multicolumn{2}{|c|}{ Floyd } & \multicolumn{2}{|l|}{ Prim } \\
\hline G1 & A - C & 2 & $A-B-C$ & 3 \\
\hline
\end{tabular}




\begin{tabular}{|c|c|c|c|c|}
\hline G2 & $A-C-D$ & 3 & $A-B-D$ & 3 \\
\hline G3 & A - D & 2 & $A-C-D$ & 3 \\
\hline G4 & $A-B-E$ & 3 & $A-C-D-E$ & 4 \\
\hline G5 & $A-B-E$ & 3 & $A-D-E$ & 3 \\
\hline G6 & $A-E-F$ & 3 & $A-C-D-F$ & 4 \\
\hline G7 & $\mathrm{A}-\mathrm{C}-\mathrm{F}$ & 3 & $\mathrm{~A}-\mathrm{D}-\mathrm{E}-\mathrm{F}$ & 4 \\
\hline G8 & $A-E-G$ & 3 & $A-B-E-G$ & 4 \\
\hline G9 & $A-E-G$ & 3 & $A-B-E-G$ & 4 \\
\hline G10 & $A-E-G$ & 3 & $A-B-E-G$ & 4 \\
\hline SCORE & 2,8 & & 3,6 & \\
\hline
\end{tabular}

Table 6 shows the ratio of the number of vertices through which the optimum path is taken; in this case, the Floyd-Warshall algorithm has an advantage with a mean value of 2.8 , and the prime has a mean value of 3.6. It means that the Floyd algorithm has a faster path in reaching the final destination because the number of vertices traversed on the optimum path is less than Prim. Comparison of the number of weights or cost of the optimum route through each algorithm can be seen in the following table.

Table 7. Comparison of total weight or cost

\begin{tabular}{|c|c|c|}
\cline { 2 - 3 } \multicolumn{1}{c|}{} & Floyd & Prim \\
\hline G1 & 6.403 & 9 \\
\hline G2 & 8.099 & 9 \\
\hline G3 & 6.403 & 7.285 \\
\hline G4 & 11.181 & 13.605 \\
\hline G5 & 9.472 & 9.857 \\
\hline G6 & 8.992 & 11.423 \\
\hline G7 & 8.261 & 8.992 \\
\hline G8 & 9.244 & 9.929 \\
\hline G9 & 9.244 & 9.929 \\
\hline G10 & 9.244 & 9.929 \\
\hline SCORE & $\mathbf{8 6 . 5 4 3}$ & $\mathbf{9 8 . 9 4 9}$ \\
\hline
\end{tabular}

The analysis results show the amount of weight or cost obtained for the optimum path through graph 1 to 2 that the Floyd-Warshall algorithm is believed to be superior to the Prim algorithm; it is seen from the average value of the Prim algorithm of 98.949 while the Floyd algorithm is 86.543 . It means that the Floyd-Warshall algorithm is more efficient in generating optimum cost routes. 


\section{CONCLUSION}

Based on the results and discussion in the previous section, there are some conclusions obtained. The Prim algorithm that is widely used to solve Minimum Spanning Tree has been proven to solve the problem of Shortest Path Problem. It is faster in determining the optimum route by generating the less active number of vertices.; it is the fastest decision-making. The Floyd-Warshall algorithm has a faster path in reaching the final destination. It is because the number of vertices passed on the optimum path is few. The Floyd-Warshall algorithm is more efficient in generating the optimum cost route than Prim. Floyd's algorithm is still better in solving the shortest path problem but slows in determining which one is the optimum route while the first algorithm is faster in determining the optimum but inefficient route and the optimum route is not the shortest and fastest path.

\section{REFERENCES}

[1] A. Putera, U. Siahaan, and R. Rahim, "Dynamic Key Matrix of Hill Cipher Using Genetic Algorithm,” Int. J. Secur. Its Appl., vol. 10, no. 8, pp. 173-180, Aug. 2016.

[2] H. Nurdiyanto and R. Rahim, "Enhanced pixel value differencing steganography with government standard algorithm," in 2017 rrd International Conference on Science in Information Technology (ICSITech), 2017, pp. 366-371.

[3] R. Rahim, M. Dahria, M. Syahril, and B. Anwar, "Combination of the Blowfish and Lempel-Ziv-Welch algorithms for text compression," World Trans. Eng. Technol. Educ., vol. 15, no. 3, pp. 292-297, 2017.

[4] R. Rahim et al., "Combination Base64 Algorithm and EOF Technique for Steganography,” J. Phys. Conf. Ser., vol. 1007, no. 1, p. 012003, Apr. 2018.

[5] R. Meiyanti, A. Subandi, N. Fuqara, M. A. Budiman, and A. P. U. Siahaan, "The recognition of female voice based on voice registers in singing techniques in real-time using hankel transform method and macdonald function," J. Phys. Conf. Ser., vol. 978, no. 1, p. 012051 , Mar. 2018.

[6] S. Aryza, M. Irwanto, Z. Lubis, A. P. U. Siahaan, R. Rahim, and M. Furqan, “A Novelty Design Of Minimization Of Electrical Losses In A Vector Controlled Induction Machine Drive," IOP Conf. Ser. Mater. Sci. Eng., vol. 300, p. 012067, 2018.

[7] A. S. Ahmar et al., "Lecturers' understanding on indexing databases of SINTA, DOAJ, Google Scholar, SCOPUS, and Web of Science: A study of Indonesians," in Journal of Physics: Conference Series, 2018, vol. 954.

[8] Cormen, T. H. Leiserson, C. E. Rivest, R. L. Stein, and Clifford, Introduction to Algorithm. United Kingdom: Cambridge, 2009.

[9] A. P. U. Siahaan, "Heuristic Function Influence to the Global Optimum Value in Shortest Path Problem," IOSR J. Comput. Eng., vol. 18, no. 05, pp. 39-48, May 2016.

[10] M. Negnevitsky, Artificial Intelligence: A Guide to Intelligent System. England: Addison-Wesley, 2005.

[11] R. Rahim, I. Zulkarnain, and H. Jaya, "A review: search visualization with Knuth Morris Pratt algorithm," in IOP Conference Series: Materials Science and Engineering, 2017, vol. 237, no. 1, p. 012026.

[12] R. Rahim, A. S. Ahmar, A. P. Ardyanti, and D. Nofriansyah, "Visual Approach of Searching Process using Boyer-Moore Algorithm," J. Phys. Conf. Ser., vol. 930, no. 1, p. 012001 , Dec. 2017.

[13] A. Singh and P. K. Mishra, "Performance Analysis of Floyd Warshall Algorithm vs 
Rectangular Algorithm," Int. J. Comput. Appl., vol. 107, no. 16, pp. 23-27, 2014.

[14] K. Srivastava and Ravikant Tyagi, "Shortest Path Algorithm For Satellite Network," Int. J. Innov. Res. Dev., vol. 2, no. 5, pp. 438-445, 2013.

[15] R. Sedgewick and K. Wayne, Algorithms, 4th ed. Addison-Wesley Professiona, 2011.

[16] D. Kalpanadevi, "Effective Searching Shortest Path In Graph Using Prim's Algorithm," Int. J. Comput. Organ. Trends, vol. 3, no. 8, pp. 310-313, 2013.

[17] S. Hougardy, "The Floyd-Warshall Algorithm on Graphs with Negative Cycles," Inf. Process. Lett., vol. 110, no. 8-9, pp. 279-281, Apr. 2010.

[18] M. Townsend, Discrete Mathematic: Applied Combinatorics and Graph Theory. The Benjamin/Cummings Publishing Company, 1987. 